\title{
La photographie chez Claude Simon : un objet mélancolique?
}

\section{Annie Clément-Perrier}

\section{(2) OpenEdition}

1 Journals

Édition électronique

URL : http://journals.openedition.org/ccs/638

DOI : $10.4000 /$ ccs. 638

ISSN : 2558-782X

Éditeur :

Presses universitaires de Rennes, Association des lecteurs de Claude Simon

\section{Édition imprimée}

Date de publication : 31 décembre 2009

Pagination : 67-83

ISBN : 9782354120580

ISSN : $1774-9425$

\section{Référence électronique}

Annie Clément-Perrier, "La photographie chez Claude Simon : un objet mélancolique ? », Cahiers Claude Simon [En ligne], 5 | 2009, mis en ligne le 21 septembre 2017, consulté le 03 mai 2019. URL http://journals.openedition.org/ccs/638; DOI : 10.4000/ccs.638 


\title{
Claude Simon :photographie, cinéma
}

\section{La photographie chez Claude Simon : un objet mélancolique?}

\author{
Annie CLÉMENT-PERRIER'
}

\section{Preuves, épreuves}

On le sait, la photographie est par essence mélancolique puisque " c'est de nous savoir mortels, ô combien, qui nous incite à fixer l'image de l'instant où nous aurons été $»^{2}$. Et toute photographie rend sensible dans un texte la dimension mélancolique du temps, atteste la vulnérabilité de l'existence, de ce qui a été, dont il reste l'empreinte lumineuse, la trace visible laissée par la chose désormais absente. Tel est du moins, à l'heure de l'avènement du numérique et de l'incertitude de son réfèrent, l'héritage de la photographie argentique qui a "pour territoire les albums, les archives ou les murs des galeries", dont chaque instantané (ou négatif-empreinte) "scelle dans sa matière les formes des choses du monde $»^{3}$. Nous savons quel rôle décisif et inspirant ont tenu dans l'imaginaire des écrivains $\mathrm{du} \mathrm{XIX}^{\circ}$ siècle, les termes du processus photographique argentique

\footnotetext{
${ }^{1}$ Auteur de Claude Simon. La fabrique dujardin, Nathan, " 128 », 1998.

${ }^{2}$ Pierre Bergounioux, Carnet de notes 1991-2000, Verdier, 2007, p. 553.

${ }^{3}$ André Rouillé, La photographie. Entre document et art contemporain, Gallimard, " Folio/ essais ", 2005, p. 616.
} 
(Гimpression, la révélation, l'imprimé, le négatif, la chambre noire, etc.) $)^{4}$ Si nous ne pouvons pas encore vraiment concevoir ce qui, dans le mode de photographie numérique, viendra influencer les modes de pensée, nous savons déjà ce qui différencie ces deux techniques de photographie, argentique et numérique. Ce sont d'abord la référence indubitable pour l'une (le " ça a existé ») puisque l'empreinte lumineuse assure une continuité de matière entre les choses, les êtres et leurs images : c'est le régime de l'empreinte, de l'analogie, de l'enregistrement de la réalité visible, de l'image comme preuve. Et la référence plus aléatoire pour l'autre (« ça pourrait être comme cela ») : il n'y a pas écriture de la lumière, mais écriture des nombres, et à la limite le référent est facultatif. Mais la différence la plus importante tient, me semble-t-il, à l'annulation d'une distance, celle du négatif et des opérations nécessaires à la révélation de l'image : tout un pan de rêverie s'en trouve effacé, et le rapport au temps et à l'espace, modifié.

On connaît la place de ce medium dans l'œuvre de Claude Simon (qui a été photographe amateur avant d'être écrivain ${ }^{5}$ ). Au regard de l'attention fascinée qu'il porte à l'acte photographique (fixer l'instant), de la similitude qui existe pour lui entre l'écriture littéraire et photographique (que souligne Denis Roche dans sa préface à Photographies. 1937-1970 $)$, et parce qu'elle valorise l'œil et la vue, l'écriture de Simon pratique, pourrait-on dire, une " photographie sans appareil $\rangle^{7}$ : elle est une écriture de la vision, une vision qui, toujours, surexpose les sensations : images à partir desquelles prend

\footnotetext{
${ }^{4}$ Voir Philippe Hamon, tout particulièrement le chapitre I (« L'image fabriquée : chambres noires ») de son essai Imageries, littérature et image au XIX' siècle, Librairie José Corti, 2007. ${ }^{5}$ Rappelons l'œuvre photographiée, Photographies. 1937-1970 (1992), Album d'un amateur (1988). Les personnages des romans témoignent aussi de cet intérêt, le narrateur du Vent (1957) prend des photos, Montès est photographe, Corinne prend des photos et les développe avec son jeune cousin dans Histoire (1967), la jeune femme de L'Acacia (1989) prend et développe elle-même ses photos.

${ }^{6}$ " C'est le rêve réalisé, à chaque livre, de tout faire tenir ensemble : mémoires des gens, mémoires des lieux, mémoires des gestes. Et une seule visée mentale occupée à tourner lentement sur elle-même, boussole sans but, développant une très lente surface de tout, grossissant l'enjeu et la métaphore, les diminuant, "fermant" l'objectif ou Couvrant" jusqu'à faire entrer tant de choses d'un seul coup dans un étrange mouvement effaré de la lumière [...] " (Denis Roche, De la ténèbre inverse, Introduction à Claude Simon, Photographies. 1937-1970, Maeght Editeur, 1992.)

${ }^{7}$ Titre d'un ouvrage de Gérard Macé, La Photographie sans appareil, Textes \& photographies de Gérard Macé, Cognac, Le temps qu'il fait, 2001.
} 
forme l'idée du roman ${ }^{8}$, façon de découper le réel (comme la mise en route de l'écriture dans La Bataille de Pharsale, avec l'éclair de l'oiseau qui raye le ciel à l'incipit, ou encore " éblouissements " de celui qui écrit dans les Géorgiques), de telle sorte que "l'esprit de l'écrivain se déploie à l'intérieur d'un minuscule espace de visée, qu'il va emplir peu à peu jusqu'à occuper toute l'immensité intérieure et rien qu'elle $»^{9}$. L'œil que Claude Simon porte sur le monde est un œil exercé à la pratique photographique, un œil toujours en quête de nouvelles voies descriptives, et l'on connaît le sort qu'il réserve à l'image $^{10}$ dans les romans des années 70 . Mais il est aussi un œil mélancolique, qui cherche à voir ce qui semble à jamais perdu de vue.

Il y a de la mélancolie dans toute photographie, disions-nous, il y en a assurément dans les photographies de l'album familial que l'on rencontre dans les romans de Claude Simon. Mais ne donneraient-elles à lire que cela ? Leur insistante présence dans la trame narrative et les variations de leur emploi, qui empruntent à l'une et à l'autre des postulations et des modalités descriptives différentes relevées plus haut, demandent que soit réinterrogée la notion même de mélancolie chez cet écrivain : ce que je me propose de faire à travers des exemples choisis dans Histoire et dans L'Herbe, deux romans qui ouvrent une perspective autobiographique dans l'œuvre.

\section{Melancholia}

C'est donc à la dimension mélancolique des photographies que scrutent intensément les narrateurs des romans simoniens à partir de L'Herbe, ce moment où Claude Simon commence à écrire à partir de son vécu, que je m'arrêterai en premier lieu. Ce que nous demandons à la photographie, c'est de fixer des moments heureux.

\footnotetext{
${ }^{8}$ À Madeleine Chapsal qui lui demande : «Vous percevez votre livre comme un instantané ? ", Claude Simon répond : "Absolument. Toutes ces choses me viennent ensemble. " Entretien réalisé par M. Chapsal "Avec Claude Simon », L'Express, n 491, 10 novembre 1960.

${ }^{9}$ Denis Roche, De la ténèbre inverse, op. cit.

${ }^{10}$ Alastair Duncan, dans son Introduction à l'œuvre de Claude Simon pour l'édition de la Pléiade, rappelle ce qui se passe dans les romans des années 70 lorsque Simon " abolit les hiérarchies en jouant avec le statut des représentations ", lorsque " une scène que l'on a crue fictivement réelle devient tableau, un tableau s'anime - pour redevenir bientôt photographie ou film. » (CE, XXXVIII).
} 
Et c'est ce qui la rend poignante lorsque les acteurs ne sont plus là. Ce n'est pas tant la photographie qui est mélancolique que le regard que nous portons sur elle, puisque dans toute photo, écrit Barthes avec son ça-a-été $e^{1}$ la mort est inscrite en creux. Sunt lacrimae rerum, il y a des larmes dans les choses, il y en a dans les photos ${ }^{12}$. Toute photographie regardée renvoie à quelqu'un, à un moment de vie fixé par l'image, désormais enfui : dans la photographie nous lisons l'absence, la perte. C'est en tout cas comme cela que nous apparaissent les photographies tirées de l'archive familiale simonienne, dont l'effet est d'autant plus efficace que nous connaissons pour avoir vu dans le Claude Simon de Lucien Dàllenbach ou dans les journaux lors des parutions des romans (en particulier L'Acacia et Le Tramway) d'émouvantes photos sépia, d'autant plus émouvantes qu'elles rendent "possible l'écriture d'une "histoire" que l'auteur se refuse à écrire $\nu^{13}$, mais qui devient de plus en plus lisible avec le temps. Le regard porté sur une photographie du Tramway sur laquelle «l'encre d'un vilain violet, $[. .$.$] comme recouverte d'un léger moisi d'un vert$ doré $[. .$.$] comme si les larmes glissant à la surface de la photogra-$ phie n'avaient entraîné qu'un peu de cette légère rouille dont étaient faits les joueurs de tennis [...] et les claires silhouettes des femmes aux grands chapeaux " (Tram., 137-138) est mélancolique. Mélancolique aussi, le regard qui s'attarde sur une photographie décrivant dans L'Acacia une jeune femme souriante « comme si le photographe avait saisi ce fugace instant d'immobilité, d'équilibre, [...] pouvant croire le temps d'un éblouissement qu'elle ne retombera jamais, qu'elle restera ainsi à jamais suspendue [...] au-dessus du vide, du noir » $\{A, 146)$.

La mélancolie n'est pas seulement présente dans les photos des romans simoniens, elle imprègne l'œuvre d'une tonalité sombre, elle est indissociable du sentiment de l'inéluctable qui affecte et nourrit l'écriture dès ses débuts. La mort, l'ombre du deuil autour des disparus hantent les romans, à mesure que se feuillettent albums et

\footnotetext{
" Roland Barthes, La Chambre claire, Gallimard, 1980.

${ }^{12}$ Je fais référence au roman de Daniel Mendelsohn, Les Disparus (Flammarion, 2007), qui cite cette phrase de Virgile, à propos de photographies qu'il regarde, seuls indices pour le narrateur, qui est aussi l'écrivain, d'existences perdues qu'il veut faire advenir par l'écriture. ${ }^{13}$ Martine Léonard, "Photographie et littérature : Zola, Breton, Simon (Hommage à Roland Barthes) », Études françaises 18, 3, Winter 1983, p. 106.
} 
archives familiales, "fatras de vieilles lettres, d'épreuves de photos et de papiers jaunis " $(V, 5)$ que ne cessent de consulter, de trâ̂ner avec eux, ou de scruter les différents narrateurs simoniens, en quête d'eux-mêmes et du passé ou de ce qui s'est passé : la ruine ou la tombe, la couleur noire, la tache, sont quelques-uns des symptômes dans l'écriture de cette melancholia, la bile noire, qui agit les êtres pour lesquels le monde est comme dévitalisé, figés qu'ils sont dans un état de deuil qui ne finit pas. Le sentiment d'étrangeté au monde en est un autre, qui se dit chez Claude Simon par le recours à la syntaxe, par les métaphores (le froid de la mort qui envahit la vieille maison familiale pour le jeune garçon qui va perdre sa mère dans Histoire, le bruit des arbres, du vent glacial, l'oncle Charles s'enfermant dans son bureau après la mort de sa femme) ou que nous rencontrons chez Montès dans Le Vent, Louise dans l'Herbe, comme séparée du monde extérieur par une vitre, chez le narrateur d'Histoire sous le poids du passé, ou le brigadier de L'Acacia sous sa cloche de verre. Le «Je ne suis rien» du mélancolique est aussi ce qui ouvre L'Herbe, le "rien » de Louise à propos de la vieille Marie occupée à mourir ("Mais elle n'a rien » [9]) s'achevant sur le " rien » (« puis plus rien»[262]) de l'auteur. Une sorte de sortilège, de sidération mélancolique rive Louise au destin de Marie ; de la même façon, le brigadier de L'Acacia augure sa mort en s'identifiant à celle de son père («Et maintenant il allait mourir " [A, 163]), comme si le mélancolique subissait et reprenait à son compte le poids ou la faute ou le destin des générations passées. Je reviendrai sur ce terme de sidération. Mais rappelons tout de suite que dans une théorie de l'image photographique conçue comme une relique, une empreinte matérielle, l'instantané apparaît comme un présent suspendu, figé, sidéré. Une vision qui renoue avec, ou prolonge, la mélancolie romaine et son désir de "fixer " l'instant, tel ce " tableau d'Antiphilos où on admirait un jeune garçon soufflant sur le feu et illuminant sous son souffle son visage. C'est un illusionnisme de l'instant. C'est un "instantané" », écrit Pascal Quignard, qui ajoute : "La vie frissonne de lumière sur fond de mort. $»^{14}$. Ou l'image pensée comme un regard «médusant » qui arrête le temps, fige l'éphémère. Or, ce qui fascine Claude Simon dans la photographie, c'est son rapport au

\footnotetext{
${ }^{14}$ Pascal Quignard, Le Sexe et l'effroi, Gallimard, 1994, p. 229.
} 
temps, c'est qu'elle est la seule à pouvoir « saisir et garder une trace de ce qui n'avait encore jamais été et ne sera plus jamais " \{Phot., avant-propos).

\section{" rousses encore sépia »}

J'en viens à Histoire, ce roman qui amorce le tournant autobiographique annoncé par L'Herbe, celui aussi dans lequel apparaissent pour la première fois ces photographies de famille que regarde ou décrit le narrateur. Mais ce qui m'intéresse ici, c'est un souvenir qui revient à la mémoire du narrateur dans ce roman d'une journée au cours de laquelle les perceptions du présent aimantent les souvenirs du passé. Il s'agit du bac dans le jardin familial dans lequel il rinçait des négatifs avec sa cousine Corinne, "si jolie penchée en avant", qui trouble le jeune garçon qu'il était alors :

[...] le bord de sa robe d'été bâillant leurs bouts rose pâle comme une enflure comme l'auréole d'un abcès se soulevant et s'abaissant respirant le bouquet de lierre au parfum de cadavre poivré terreux dans l'air surchauffé Disant ça suffit maintenant il n'y a qu'à les laisser sous le robinet tu les surveilleras Sa main cessant d'agiter l'eau du bac les reflets cessèrent de se mélanger se regroupèrent d'un côté le ciel de l'autre la touffe de lierre renversée noire son bord dentelé festonné divisant le bac à peu près en deux parties Dans l'une je pouvais voir les nuages éblouissants glisser et dans l'autre (celle où se reflétait la masse obscure du lierre) les petits rectangles tachetés sépia couleur de feuilles mortes comme de minces pellicules de temps de l'été mort auquel nos images (certaines floues et bougées comme si nous étions passés devant l'appareil clignant des yeux dans le soleil à une vitesse terrifiante et vertigineuse) restaient accrochées [...] (Hist., 92-93)

On aura noté la dimension photographique de la scène : le bac et le bain révélateur, les images, les "pellicules de temps », le clair et l'obscur, la nébuleuse photochimique (les nuages, la lumière). Ces négatifs seront évoqués une deuxième fois dans Histoire (p. 379 et 380 ), et c'est encore par la médiation de Corinne et la vision qu'il en avait dans son enfance et son adolescence (p. 109, 116, 120, 122) que le narrateur est amené à se souvenir d'une fontaine alimentant un bassin rempli d'une eau noire et de feuilles pourrissantes lui rap- 
pelant les pellicules sépia, lors d'une visite d'un musée en Grèce avec Hélène qu'il est sur le point de quitter. L'image de l'une se superposant à l'autre, le désir trouble que Corinne suscitait alors éveillant d'autres évocations de trouble et de désir, de sexualité pleine de culpabilité devant le désarroi d'Hélène. Mort de l'amour et sexualité, mélancolie et désir d'aller vers autre chose, inextricablement liés ici :

Dans les parties du bassin où l'eau reflétait les feuillages sombres des lauriers on en voyait d'autres accumulées dans le fond vert-noir brunes visqueuses pourrissant les plus récentes rousses encore sépia collées ensemble par paquets minces pellicules de temps d'été mort invisibles dans la moitié du bac que remplissait le ciel les nuages éclatants mais après la découpe dentelée de la crête du buisson de lierre on pouvait distinguer nos images quelques-unes floues comme si nous étions passés devant l'objectif emportés à toute vitesse (Hist., 380)

Je reviendrai sur cette séquence, mais il me faut pour l'instant m'arrêter sur la métaphore de la photo/feuille morte (" rousses encore sépia »), métaphore mélancolique et classique des humains comme « des feuilles, qui tantôt vivent pleines d'éclat, en mangeant le fruit de la terre, et tantôt se consument et tombent au néant $»^{15}$. Et dans $L a$ Corde raide, n'est-ce pas la feuille qui se détache de l'arbre et glisse dans l'eau, qui vient à l'esprit à la lecture de ces lignes sombres sur la mort et la façon dont les mourants semblent «se détacher de vous avec indifférence, vous repousser, et s'enfoncer en eux-mêmes au moment où ils ont compris que l'heure de leur mort est arrivée " $(C R, 21)$ ? Dans L'Herbe, des carnets de Marie que regarde Louise, glisse une photographie " elle-même semblable - l'épreuve au bromure mal fixé, jaunie, aux reflets bruns - à une feuille morte, avec son assemblée de personnages morts posant »\{Herbe, 223-224). Une feuille morte destinée à pourrir, comme les corps des personnages (« presque tous étaient morts») posant avec nonchalance sur une ancienne photo du mariage de Pierre et Sabine (69) étaient destinés à bientôt " pourrir, souvent sans sépulture, ou hâtivement recouverts de quelques pelletées de terre, ou jetés pêle-mêle dans un charnier avec ceux des milliers, des centaines de milliers de leurs 
semblables » (72). Je souligne le détail de cette photo/feuille morte, car la feuille pourrissante, le tapis de feuilles pourries est quelque chose qui revient dans l'œuvre, et en particulier dans Les Géorgiques et LeJardin des Plantes ${ }^{16}$. Cette photographie glissant des carnets de Marie comme une feuille morte est à rapprocher, dans un passage des Géorgiques, de la feuille que regarde le visiteur, "se détachant, tombant de branche en branche en molles glissades, se balançant dans l'air immobile comme une plume d'or terni, impondérable, éphémère, s'affalant enfin sans bruit » $(G, 162)$ avant de rejoindre le " tapis pourrissant » de feuilles pourrissantes, ce " magma végétal " à partir duquel repoussent des « touffes d'herbe d'un vert paradoxalement tendre " (163), par les lois de " transmutation " (162) de la matière (" l'air humide, frais, chargé d'un subtil et délétère parfum non de mort, d'agonie, de putréfaction : plutôt (comment dire ?) de transmutation ». La mélancolie profonde du spectacle de la mort et de la pourriture dans la nature cède place à l'idée que la vie est plus puissante et que les formes naissent à partir d'autres formes ou sous d'autres formes. Ce qui est mort continue à vivre autrement. Comme si le regard porté par Simon sur le cycle cosmique, le retour des choses si sensible dans tous ses romans, était dicté par un néces-

\footnotetext{
${ }^{16} \mathrm{Il}$ y a le souvenir du bassin tapissé de feuilles pourries du Jardin des Plantes dans lequel S. enfant manque se noyer : dans leur article "Les feuilles au fond du bassin. Sur LeJardin des Plantes de Claude Simon" (L'Infini, $\mathrm{n}^{\circ}$ 65, printemps 1999, p. 88-111, p. 88), Patrick Longuet et Jean Marc Dilettato voient "son livre, remontant à l'enfance " procéder " à un découvrement qui révèle un souvenir-écran, donnant à voir un "fond du bassin tapissé d'une litière de feuilles mortes rousses brunes rouille prune pourries gluantes sous ses mains" » (JP, 1167). Il y aussi la feuille que S. regarde tournoyer (1103), ou qui s'immobilise (1125), ou la feuille de Kirilov (943), cette feuille d'automne un peu pourrie sur ses bords que Kirilov enfant, en fermant les yeux, se représentait encore « verte, brillante, avec ses nervures, sous le soleil », et, dit-il « ce que j'avais vu était trop beau » et n'était plus la réalité. Une feuille, dit-il encore à Stavroguine, " est une feuille, tout simplement ». Cette simple feuille est quelque chose qui porte la rêverie, non pas seulement celle de Kirilov, mais celle de Claude Simon.
} 
saire désir de " donner une chair à l'ombre $»^{17}$, était dicté par un désir de s'arracher à l'entreprise de sidération, de fascination sensible chez bien des personnages de romans simoniens devant la mort, de puiser une ressource dans la beauté du monde et le cycle de la nature. On se souvient du brigadier de L'Acacia échappé du camp de prisonniers, qui se réconcilie avec l'humain après avoir recopié l'écriture des herbes, des feuilles, des cailloux. Et l'on sait à quel point Claude Simon est fasciné par ce que Merleau-Ponty appelle " la chair du monde ", à quel point son écriture rend palpable cette fascination, à quel point elle est désir de rendre compte de cette présence au monde. Alors, dans cette leçon de vie des Géorgiques qui fait appel au subtil travail de transformation de la matière, il semble que l'on puisse lire quelque chose qui ressemble au travail de transformation de l'écriture sur la matière mélancolique du passé, et cela tout particulièrement dans Histoire, ce roman dans lequel il est question de distillation, de sublimation de la matière, dans la chambre noire ${ }^{18}$ aux volets clos de l'oncle Charles. Quelque chose qui ressemble au travail auquel celui-ci se livre et qui évoque également l'évolution du développement photographique, puisque c'est par le biais "d'une série de transformations techniques, matérielles et formelles qu'un phénomène réel devient un phénomène photographique [...] chaque étape se caractérise par un changement de matière : une transsubs-

\footnotetext{
${ }^{17}$ Cette idée est développée par Roland Gori à propos des cadavres déchiquetés par les rapaces lors des rites funéraires en Inde, dans lesquels une analysante puise une sorte de consolation : "En somme », écrit-il, " la perte continuerait de nourrir la vie dans un cycle cosmique qui pacifie la douleur ? la jeune femme analysante aurait ainsi rencontré dans le souvenir du documentaire un fantasme inscrit dans sa mémoire : donner une chair à l'ombre en se nourrissant de la perte. Le désir se nourrit de cette perte. " (Logique des passions, Flammarion, 2005, p. 70). Est-ce ce qui fascine S. dans Le Jardin des Plantes (p. 150 et 252) lorsqu'il rappelle les rites funéraires en Inde et qu'il y revient à plusieurs reprises dans le roman? Je suis tentée de le croire.

${ }^{18}$ Il y a dans l'œuvre de Claude Simon bien des chambres noires ou chambres d'écriture, dans des romans tels qu'Histoire, La Bataille de Pharsale, L'Acacia, Le Jardin des Plantes, ou encore la chambre d'hôtel de La Route des Flandres dans laquelle Georges tente de mettre en mots pour Corinne son expérience de la guerre.
} 
tantiation $»^{19}$. Image photographique ou image végétale ${ }^{20}$, ce qui s'énonce là touche à la peau, à la chair comme à la feuille du livre, et touche au pouvoir du roman de mettre à distance les expériences vécues en les incarnant dans les mots.

Histoire, seul roman dans lequel il est question de négatifs, d'images en cours de développement, en devenir, donne donc à lire dans les images doublement muettes ${ }^{21} \mathrm{du}$ bac et de son bain révélateur ce qui va être entrepris par Claude Simon à partir de ce moment-là, qui ajoute aux recherches formelles une dimension autobiographique ${ }^{22}$. Une dimension autobiographique à mettre entre guillemets, en partie écrite à l'insu du narrateur (l'eau noire dans une partie du bac, le passé, la feuille pourrissante, ce qui a eu lieu), contredite par l'eau claire dans l'autre partie, les variations des nuages et les jeux de la lumière qui s'y reflètent, à l'image même du cadre de la page et du dessein infini et mouvant de l'écriture qui en jaillira, dont il ne sait encore rien. Tous éléments venant dire à la fois la mélancolie et le mouvement dynamique des recherches : identitaire, familiale, et formelle, que concentre le titre du roman, Histoire, istoria, quête, enquête. N'est-ce pas sa propre présence que questionne, à travers les photos parentales d'Histoire, ce « Moi ?...» du narrateur à la toute fin du roman?

Il y a bien une dimension mélancolique et intimiste dans l'évocation des photos rincées dans un bac : les images, "nos images (certaines floues et bougées ») appellent tout de suite un rapprochement avec le « halo flou » qui entoure le portrait du père (qui n'est

\footnotetext{
${ }^{19}$ A. Rouillé, op. cit., p. 118-119.
}

${ }^{20}$ Dans sa communication «"Dans la lumière rouge du laboratoire". La métaphore comme révélateur du lieu chez Claude Simon », Jean-Yves Laurichesse rappelle, à l'heure de la photographie numérique, qu'une " pellicule photographique noir et blanc est constituée d'une feuille de matière souple" (Transports. Les métaphores de Claude Simon, études réunies par Irene Albers et Wolfram Nitsch, Peter Lang GmbH, Frankfurt am Main, 2006, p. 220).

${ }^{21}$ Philippe Hamon : " Image muette, la photographie est aussi comme le "négatif" de l'image littéraire parlée ou écrite. Et ce négatif dit peut-être quelque chose, toujours, plus ou moins indirectement, sur la littérature, qui elle-même est, bien souvent, un mode indirect, ou inversé, ou allusif, ou "négatif", ou ironique [...] de refléter le monde. " \{op. cit., chap. I, p. 59).

${ }^{22}$ Dominique Viart souligne, au chapitre "Variations autobiographiques » de son ouvrage, les trois notions qui en composent le nom, car cette dimension autobiographique est une recherche " qui interroge à la fois la vie, le sujet et l'écriture » (Dominique Viart et Bruno Vercier, La Littérature française au présent, Héritage, modernité, mutations, Bordas, 2005, p. 61). 
pas nommé comme tel dans le roman) sur la photographie (toujours dans Histoire) que le narrateur regarde lorsqu'il est enfant, " le fond sépia clair allant pâlissant en dégradé jusqu'au blanc de sorte qu'il avait l'air de planer [...]»\{Hist., 17), venant moins parler de mélancolie et d'un père inconnu que de l'ouverture de cette image à la dimension du mythe (repris par la majuscule, la déification, la souffrance du Christ dans le texte). Les choses sont dites en filigrane, à l'encre sépia, ce nuage d'encre dans lequel se dérobe la seiche en fuyant. Ce qui nous est aussi donné à lire, c'est ce reste (A. Rouillé) qui accompagne l'acte photographique, qui flotte autour de l'image, ce trouble auquel se mêle la mort toujours présente dans la vision de l'auteur (l'été mort, l'odeur de cadavre du lierre, etc., lefascinum, cet effroi dont parle Pascal Quignard), dont il se souvient à la vue des feuilles mortes évoquant les clichés dans l'eau. Il y a le temps de la prise photographique (le jeune garçon et sa cousine, donc le présent vécu), dans lequel prend place l'image au moment de sa saisie avec toutes les sensations qui l'accompagnent. Et puis, il y a le temps des clichés encore flous, de l'image pas encore là et pourtant déjà là, le temps de l'image latente, ouverte sur le devenir. Et ce qui est sensible dans les images " comme si nous étions passés à toute vitesse", c'est le passé qui déjà envahit corps et choses : encore là etpourtant déjà passés alors même que la photo n'est pas encore développée ${ }^{23}$. Et pour celui qui se souvient, le présent se mêle de passé et de son présent contemporain, montrant qu'un moment photographique ne se réduit pas seulement à la photographie qui sera regardée, mais fait intervenir tout ce qui se produisit alors et qui n'était pas entièrement là, c'est-à-dire perçu comme tel. Par ailleurs, comme dans le moment de l'écriture, ce moment de perception au présent qui s'accompagne de mémoire, réelle, incertaine, lacunaire, fictive, ne pouvant jamais être confronté à ce qui est représenté, peut se prêter à toutes sortes de manipulations. On s'en souvient à propos de la "photo de l'atelier", toujours dans Histoire, à propos de "l'image fuligineuse (non pas floue si l'on regarde plus attentivement, mais bougée) » (267) qui a été " inventée de toutes pièces à partir d'une

\footnotetext{
${ }^{23}$ Je m'inspire ici du chapitre "Temporalités photographiques» de l'ouvrage d'André Rouillé, déjà cité, p. 276.
} 
photo parfaitement nette $»^{24}$ dont Claude Simon visualise par un dessin la mise en mouvement, pour suggérer (et brouiller tout à la fois) l'image du transfert du neveu sur l'oncle ou de l'oncle sur le neveu. Le flou photographique prend ainsi en charge le mystère narratifengendré par les glissements qui s'opèrent dans le texte.

Mais il y a bien autre chose encore, qui touche au dispositif formel : le rappel des photos/feuilles mortes dans Histoire inaugure l'intertextualité fameuse et largement commentée des feuilles de l'arbre devant la fenêtre à l'incipit d'Histoire et à la clausule de L'Acacia, des feuilles animées par le souffle du vent et celui de l'écriture. Ce que Claude Simon rend sensible au lecteur, par la récriture des photos/ feuilles mortes, c'est de la durée, du temps non pas figé, fixé, mais du temps de latence, du roman, de l'écriture, de la lecture, dont il (le lecteur) peut percevoir l'épaisseur palimpseste. Et puis, ces images "floues ", ne marqueraient-elles pas, bien avant l'heure du numérique, l'intention d'un regard sur l'image en elle-même plutôt que sur son référent?

\section{La photo dans le roman, le roman d'une photo}

L'image photographique est sans doute traversée par deux grands modes, l'un affirmatif (le " ça-a-été », l'empreinte du passé), et l'autre interrogatif (« que s'est-il passé ? »), qui est sans réponse, qui ouvre donc sur un champ d'incertitude, d'oublis, de virtualités, de secret, au fondement même de la mémoire, de la manipulation, de l'écriture. Mais à cela il arrive que Claude Simon ajoute la question autrement troublante et déconcertante du « qu'aurait-il pu se passer? ", comme on va le voir avec la photographie de L'Herbe.

Revenons en effet à la photographie qui s'échappe des carnets de Marie, la vieille femme en train de mourir, cette photo que ramasse et scrute la jeune Louise sur le point de quitter son mari, Georges, qu'elle croit d'abord reconnaître enfant, mais elle s'aperçoit, puisque la photo est datée (août 1896), qu'il s'agit de Pierre, le père de celuici. Toute photo met en évidence la ressemblance, la filiation, l'identité, l'héritage en quelque sorte, et c'est précisément cette photo de L'Herbe qui annonce, bien avant Histoire, je le crois, l'entreprise au-

\footnotetext{
${ }^{24}$ C'est ce que nous apprend Lucien Dallenbach dans les notes (40) de son Claude Simon, Editions du Seuil, « Les contemporains », 1988, p. 203.
} 
tobiographique qui s'écrit en filigrane des recherches formelles et scripturales mises en œuvre dans Histoire.

Mais autre chose est à lire dans la photo regardée par Louise dans L'Herbe. Ce n'est plus Georges ou Pierre que regarde Louise, mais Marie jeune fille et le jeune botaniste au sujet desquels elle invente une histoire qui n'a pas eu lieu :

[...] et peut-être, un peu plus tard, les deux silhouettes - la robe de bronze et celle en culotte cycliste - glissant lentement, déchiquetées, entre les branches croulantes du verger évanescent (comme si Louise avait pu les voir, les suivre des yeux, [...] et [...] tous les deux, secouant l'arbre, riant - ou plutôt le prunier secoué, le froissement de feuilles, sans que l'on voie autre chose qu'un fragment de robe ; une main, un bras ? - [...] lui faisant sa demande, demandant la permission de revenir, et elle disant Oui en le regardant bien en face, de ses yeux clairs, tranquilles [...]. (Herbe, 233-234)

Ce faisant, "Louise invente, devant la photographie de Marie jeune, le "roman" d'un amour sacrifié qui donne un sens à la vie qui s'achève. La lecture qu'elle fait de la photographie projette évidemment sa propre angoisse et la photo devient alors l'emblème du travail du romancier $\|^{25}$. La photographie est une "machine à fictions" (Anne-Marie Garat), sans aucun doute ${ }^{26}$. Et photographie et écriture ont un même potentiel de virtualité ${ }^{27}$. Ici, la photographie n'est plus un embrayeur de souvenirs, elle met en mouvement quelque chose qui aurait pu être et qui est de l'ordre du désir. Louise regardant les deux personnages, Marie et le jeune botaniste, met en scène son désir de faire vivre quelque chose qui n'a pu avoir lieu, peut-être resté dans les limbes ou qu'elle imagine, et ce désir né de la mélancolie du « jamais plus », je me risque à l'appeler une mélancolie désirante. Mais

\footnotetext{
${ }^{25}$ Martine Léonard, art. cit. p. 106.

${ }^{26}$ On pourra se reporter à son essai Photos defamille, Seuil, 1994. Notons aussi l'album de photo comme roman familial, la photographie comme lien entre le fait vécu et l'art, ou la photographie comme écriture narcissique, comme carnet de voyage, comme journal intime, comme corpus de travail et de recherche de l'œuvre à venir, autant d'approches de la photographie comme outil intimiste et créatif pour bien des artistes, dans la communication d'Elvire Perego, "Intimités et Jardins secrets, l'artiste en photographe amateur ", Nouvelle Histoire de la photographie, Bordas/Adam Biro, 1994, p. 335-345.

${ }^{27}$ Rappelons avec A. Rouillé que « Le virtuel désigne ici ce qui n'existe qu'en puissance et non en acte. " (op. cit., p. 263). Voir aussi Pierre Lévy, Qu'est-ce que le virtuel ? La Découverte/Poche, «Essais », 1998.
} 
il y a une différence sensible entre le travail de reconstitution d'une vie au plus près de la réalité, autour d'une photographie, telle l'entreprise de Pierre Bergounioux dans Miette, par exemple, qui tente de retrouver par l'écriture un personnage disparu, et l'entreprise de Claude Simon dans L'Herbe, qui transforme une donnée (la photo) en variable, en quelque chose autre, quelque chose qui échappe un instant à son destin, qui transforme ce qui a-été en ce qui aurait pu être. Le récit s'envole vers autre chose, et la mélancolie autobiographique, dans ce traitement si spécifique de l'image par où le " c'est ainsi » devient " cela pourrait être comme cela », cède place à autre chose qui en appelle plus à la retouche numérique qu'à l'inscription du passé ou du réel, suggérant ou anticipant ainsi par bien des aspects l'image virtuelle $\mathrm{e}^{28}$. La photographie est ainsi soustraite à son statut de relique, et arrachant Marie à son destin de vieille fille, Louise s'arrache elle-même à une contemplation médusante, à sa propre mélancolie. Le mélancolique tel que le définit la psychologie ne désire plus rien, ne souhaite plus rien, reste prostré sans plus envisager d'avenir, alors que celui qui écrit ne cède pas au destin ou aux forces obscures qui se sont emparées de lui, mais trouve dans la nécessité vitale qui le pousse à arracher aux ténèbres du passé ce à quoi il veut avoir accès, ce qu'il veut faire advenir au jour, de quoi nourrir un puissant désir de vivre. Louise " comme morte " dans l'herbe, trouvant dans les carnets de Marie matière à vivre et matière à écrire, le brigadier de L'Acacia échappé du camp de prisonniers se réconciliant avec l'humain après avoir recopié l'écriture des herbes, des feuilles, des cailloux, traduisent dans le texte les forces vives auxquelles s'attelle l'écrivain dans son désir de s'arracher à celles qui le tirent vers le néant.

\footnotetext{
${ }^{28}$ C'est ce qu'a bien vu Philippe Ortel lorsqu'il écrit à propos des cartes postales d'Histoire, que « les contenus de réalité offerts par les images sont manipulés et deviennent la matrice de développements imaginaires dont l'effet est de virtualiser le référent, non d'exalter l'inscription de son existence dans le temps, comme le fait la photographie ordinaire. " ( L'invention du virtuel. Claude Simon et la photographie », Les Images chez Claude Simon. Les mots pour le voir, La Licorne, $n^{\circ} 71,2004$, p. 168. Voir aussi Jean Rousset, "Histoire de Claude Simon. Le jeu des cartes postales », Versants, ${ }^{\circ} 1$, Lausanne, Neuchâtel et Genève, 1981, p. 121-133.
} 


\section{Une mélancolie désirante}

J'ai beaucoup parlé du désir qui est, comme l'on sait, ce qui se tend vers un but jamais atteint, jamais satisfait. J'ai parlé du désir de Louise d'arracher Marie à son destin et à la lourdeur de la fatalité, j'ai parlé du désir né du " jamais plus », j'ai employé cette expression de " mélancolie désirante », et il est temps maintenant d'aller regarder du côté de la mélancolie simonienne, de la définition très particulière qu'en donne Claude Simon dans Le Jardin des Plantes à plusieurs reprises par la bouche de S., lorsque le journaliste l'interroge sur ce qu'il éprouve au moment de l'embuscade, et qu'il s'exprime à propos du roman de Dostoïevski (L'Idiot) et de la "déchirante et mélancolique avidité avec laquelle le condamné regarde autour de lui le monde [...] qui va continuer à exister alors que dans quelques instants lui-même ne sera plus rien " $(J P, 1124)$. Il s'oppose ainsi à Stendhal dans sa description de Julien Sorel marchant à la guillotine et qui éprouve une sensation délicieuse en marchant au grand air :

Bon Dieu! raconter que marcher au grand air est pour quelqu'un qui va mourir une "sensation délicieuse ", bon Dieu! le soleil, les oiseaux, le vert des prés, le bleu du ciel, l'air doux à respirer! [...] et S. disant que Non ce n'était rien de tout ça, qu'il y aurait peut-être un mot, mais qu'on lui donne en général un sens qui Hésitant [...], et à la fin il dit Mélancolie, le journaliste s'exclamant Mélancolie !... (JP, 1119-1120)

Puis plus loin, il continue, disant que cette mélancolie était exactement le contraire de ce qu'on imagine habituellement, non pas une songerie, mais « quelque chose de violent qui protestait, furieux, bâillonné mais hurlant: Jamais je n'avais tant désiré vivre, jamais je n'avais regardé avec autant d'avidité, d'émerveillement, le ciel, les nuages, les prés, les haies " (1122).

Cette notion ambiguë, difficile à saisir, puisqu'elle s'oppose à la définition classique de la mélancolie, cette mélancolie-là est une mélancolie désirante, parce qu'elle exprime tout à la fois le violent regret des choses, de ce qui va être perdu, et qu'elle est intimement liée au désir violent de vivre qui fait irruption au moment du faceà-face avec la mort brutale, inattendue. Un désir qui arrache à la fatalité que le mélancolique traîne avec lui, un désir qui n'entre pas 
vraiment dans le champ désolé du déprimé mélancolique pour qui le monde est désincarné. Il y a dans cette « déchirante et mélancolique avidité » un violent désir de capter l'instant, de l'arrêter, d'en prendre une sorte d'instantané( « le ciel, les nuages, les prés, les haies »), de se sentir pris encore dans le mouvement du monde. Dans l'intensité et le sursaut de l'instant par lequel le mélancolique échappe aux figures de sa mélancolie pour se ruer vers la vie, on retrouve le désir de la mélancolie romaine de travailler la terreur de la mort par la fureur de vivre l'instant. Et dans l'oxymoron simonien (« mélancolique avidité »), on retrouve les effets d'un verbe, du verbe désirer qui, dans le de- privatifde ce verbe issu du desiderare latin (ce verbe de la sidération) laisse entendre le regret (l'absence, la perte) et l'arrachement (à la contemplation), si l'on oublie le champ psychanalytique du désir pour retrouver l'étymologie ancienne ${ }^{29}$. Mélancolie et désir sont liés dans une même figure du regret. Et l'on retrouve les deux versants de ce verbe désirer (qui prend tardivement la valeur positive que nous connaissons) dans l'écriture même de Claude Simon, dans la sidération mélancolique ou le ressassement de certains aspects de sa vie, et sa capacité à s'arracher à cette tentation mélancolique, à " dépsychologiser " en quelque sorte les affects du deuil, de la mort, pour mettre en place autre chose qui est de l'énergie créatrice. L'énergie qui sous-tend l'écriture est aussi un effet de la mélancolie, un effet de l'inquiétude qui travaille l'œuvre, l'angoisse du mot juste, de la sensation indicible, le sentiment de perte ou de manque quant au but jamais tout à fait atteint, jamais tout à fait réalisé. Pour un écrivain comme Simon qui ne travaille pas sur une idée, mais à partir des mots et de la mémoire, de la perception active, interrogative, l'écriture est une source d'inquiétude et d'insatisfaction permanentes. Cette mélancolie-là entretient le feu du désir d'écrire, car l'insatisfaction permanente et le ressassement qui marquent l'écriture telle que la pratique l'écrivain toujours dans le doute de ce qu'il fait, sont aussi désir de vouloir toujours plus et autre chose, de faire toujours retour sur ce manque, cette perte infligés par la mémoire et les sensations

\footnotetext{
${ }^{29}$ Le verbe désirer, venu du desiderare latin, composé du de- (à valeur privative) et de sidus, -eris, " astre» (sidéral, sidéré), dans sa forme latine signifie littéralement « cesser de contempler » (l'étoile, l'astre), d'où moralement " constater l'absence de », avec une forte idée de regret. L'adjectif sidéré est emprunté au participe passé sideratus, du latin impérial siderari " subir l'action funeste des astres ».
} 
justes, que l'écriture tente de combler. Et cette mélancolie-là entretient la tension $^{30}$ qui habite l'écriture de Claude Simon. Lorsqu'on lui demande ce qui le captive dans l'image et dans l'acte photographiques, il parle de la capacité de la photographie à « fixer, retenir une image de ce présent dont parle Borges et qui se désintègre à tout instant dans le passé, et aussi la recherche d'une certaine beauté en soi, d'harmonies $\|^{31}$ : c'est dans cet écart entre les réflexions de l'écrivain mêlant si intimement (ici à propos de la photographie) la vie et l'art, entre l'emprise qu'elle a sur lui et le traitement qu'il lui offre dans les romans, que prend naissance cette sorte de tension que je soulignais ici. Une tension permanente, jamais résolue, toujours à l'œuvre, mais qui, peut-être, parvient à arracher l'écriture à partir de la photographie, à la seule mélancolie de la trace et de l'empreinte du passé.

\footnotetext{
${ }^{30}$ Tension que souligne Dominique Viart dans sa communication «Une écriture nomade. La puissance critique de la métaphore simonienne. " L'écriture de Claude Simon, note-t-il, est constamment « en tension, disponible mais surveillée, offerte à la dérive ou à la pente des rêveries verbales mais exigeante et critique avec elle-même. " (Transports, les métaphores de Claude Simon, op. cit., p. 16). Ce style si particulier de Claude Simon, il le définissait déjà dans Une mémoire inquiète (PUF, 1997) comme « une poétique de l'épanorthose» (p. 259).

${ }^{31}$ Photographies Magazine, n 40, «Une écriture en noir et blanc », avril 1992.
} 